\title{
Robust control of systems with output hysteresis and input saturation using a finite time stability approach
}

\author{
Damiano Rotondo, Gianluca Rizzello, Member, IEEE, David Naso, Senior Member, IEEE
}

\begin{abstract}
This paper presents a robust control approach for a class of nonlinear dynamic systems consisting of a linear plant connected in series with a hysteresis operator, and affected by control input saturation. Such a class of systems commonly appears in applications concerning smart materials, in particular thermal shape memory alloys wire actuators. The goal of this paper is to design a robust controller, in the form of an output PI law, which ensures set-point regulation with a desired decay rate and, at the same time, accounts for the effects of both hysteresis and input saturation. The resulting controller appears as attractive on the implementation standpoint, since no accurate hysteresis compensator is required. In order to deal with the proposed problem, the hysteretic plant is first reformulated as a linear parameter-varying system. Subsequently, a finite time stability approach is used to impose constraints on the control input. A new set of bilinear matrix inequalities is developed, in order to perform the design with reduced conservatism by properly exploiting some structural properties of the model. The effectiveness of the method is finally validated by means of a numerical case of study.
\end{abstract}

Keywords: Hysteresis, saturation, finite time stability (FTS), linear parameter-varying (LPV) system, bilinear matrix inequalities (BMI), robust control.

\section{INTRODUCTION}

Smart materials represent an effective means to design micropositioning systems capable of accuracy, efficiency, and lightness not achievable with conventional actuation technologies. Some examples of smart materials commonly used in mechatronic actuators include thermal and magnetic shape memory alloys, piezoelectric ceramics, and electroactive polymers [1], [2].

Despite their many advantages in terms of performance, smart material systems often exhibit a highly nonlinear and rate-dependent behavior which significantly complicates their modeling and control. In particular, the most common

This work has been partially funded by the Spanish State Research Agency (AEI) and the European Regional Development Fund (ERFD) through the project DEOCS (ref. MINECO DPI2016-76493), by AGAUR of the Generalitat de Catalunya through the Advanced Control Systems (SAC) group grant (2017 SGR 482) and by the Spanish State Research Agency through the Maria de Maeztu Seal of Excellence to IRI (MDM-2016-0656) and the grant Juan de la Cierva - Formacion (FJCI-2016-29019).

D. Rotondo is with the Institut de Robòtica i Informàtica Industrial, CSIC-UPC, Llorens i Artigas 4-6, 08028 Barcelona, Spain, and with the Research Center for Supervision, Safety and Automatic Control (CS2AC) of the Universitat Polit'ecnica de Catalunya (UPC). e-mail: damiano.rotondo@yahoo. it

G. Rizzello is with the Department of Systems Engineering and the Department of Material Science and Engineering, Universität des Saarlandes, 66123 Saarbrücken, Germany. e-mail: gianluca.rizzello@imsl.uni-saarland.de

D. Naso is with the Department of Electrical and Information Engineering, Politecnico di Bari, 70125 Bari, Italy. e-mail: david.nasoepoliba.it nonlinearity which appears in such systems is represented by hysteresis [3]. In case the control system does not explicitly account for the effects of hysteresis, severe degradation in accuracy, or even instability, may occur. In recent years, a number of contributions have performed systematic analysis and design of control systems with hysteretic nonlinearities. The most popular control approach consists of using an inverse compensator based on an accurate mathematical model of the hysteresis, e.g., the Prandtl-Ishlinskii model [4] or the Krasnosel'skii-Pokrovskii model [5]. Eventually, the robustness of such strategies can be furthered by combining the conventional hysteretic compensator with adaptive control [6], [7]. However, due to the high complexity of the resulting models, hysteresis compensation turns out to be highly demanding on a numerical standpoint. For this reason, a number of authors have investigated analytical tools which permit to address the control of hysteresis without performing an explicit compensation. Some examples include passivity-based approaches, [8], [9], integral controllers [10]-[13], servocompensators [14], and sliding mode control [15]. The main advantage of these approaches is the significant reduction in computational complexity of the resulting controller, which might be preferable in some applications.

In addition to hysteresis, smart material actuators are also often affected by control input saturation, due to physical limits of the corresponding control variables (e.g., maximum heating power, electric field, or magnetic field sustainable by the material). Since in many cases one is interested in maximizing the system performance, the materials are often operated close to their limits. Therefore, control input saturation becomes also a critical aspect for the design of control system for smart materials. It is remarked how the analysis of control systems with saturation has been performed by a number of authors, and different solutions have been developed to deal with such a nonlinearity, see, e.g., [16]-[19].

Despite many authors have investigated control of systems exhibiting hysteretic and saturation nonlinearities separately, the control of system characterized by simultaneous saturation and hysteretic nonlinearities has not received a significant attention so far. We remark that being able to account for the effects of both hysteresis and control input saturation is fundamental for the correct exploitation of the high potentials of smart materials in closed loop architectures.

The main contribution of this paper is the development of a robust, compensator-free control system for a class of nonlinear systems with input saturation, consisting of a cascade 
of a linear dynamics and a hysteresis. This class of systems is representative of the behavior of thermal shape memory alloys wire actuators, which are commonly described by a first order transfer function, which models the thermal activation of the material, cascaded with a rate-independent hysteretic operator, which describes the material-dependent temperature-deformation characteristic [20]-[22]. An example of typical temperature-displacement hysteresis of a shape memory alloy wire-spring actuator is depicted in Fig 1. In this work, the goal is to regulate the system output to a desired set-point, while ensuring some dynamic performance with respect to hysteresis and avoiding, at the same time, control input saturation. First, based on a result presented in [23], the initial hysteretic model is converted into a linear parameter-varying (LPV) system in which the hysteresis is represented by a bounded time-varying gain [24], [25]. For such a new system, a PI law is proposed in the form of a time-varying linear combination of states. Such a control law is attractive for implementation purpose, since it does not rely on a computationally involved hysteresis model. The control input of the reformulated LPV system is given by the time derivative of the control input of the original system, therefore standard LPV theory cannot be applied to deal with control saturation [19]. To overcome such a limitation, a novel approach is proposed based on the concept of finite time stability (FTS) [26], [27]. A novel set of bilinear matrix inequalities (BMI) is developed to address the controller design, by ensuring the following closed loop specifications: the system output asymptotically tracks a constant set-point with a desired exponential decay rate $\alpha<0$; the control input never saturates; robust stability and performance with respect to the hysteresis; the model structure is properly exploited in order to reduce design conservativeness. Numerical validation of the method is finally presented by means of a simulation case of study.

The remainder of this paper is organized as follows. The control problem is first introduced in Section II. The main result, i.e., the development of the BMI conditions which allow to define the design problem, is presented in Section III. Simulation validation of the approach is performed in Section IV. Finally, conclusions and future developments are discussed in Section V.

\section{PROBLEM FORMULATION}

Let us consider a linear time invariant (LTI) single-inputsingle-output (SISO) system subject to actuator saturations:

$$
\begin{aligned}
\dot{x}(t) & =A x(t)+b s a t(u(t)) \\
y(t) & =\Gamma(c x(t))
\end{aligned}
$$

where $x \in \mathbb{R}^{n_{x}}$ is the state, $u \in \mathbb{R}$ is the control input, $y \in$ $\mathbb{R}$ is the measured output, $A, B, C$ are matrices (or vectors) of appropriate dimensions, $\Gamma(\cdot)$ is a hysteresis operator and sat $: \mathbb{R} \rightarrow \mathbb{R}$ is the saturation function, defined as:

$$
\operatorname{sat}(u(t))=\left\{\begin{array}{lc}
\bar{u} & u(t)>\bar{u} \\
u(t) & |u(t)| \leq \bar{u} \\
-\bar{u} & u(t)<-\bar{u}
\end{array}\right.
$$

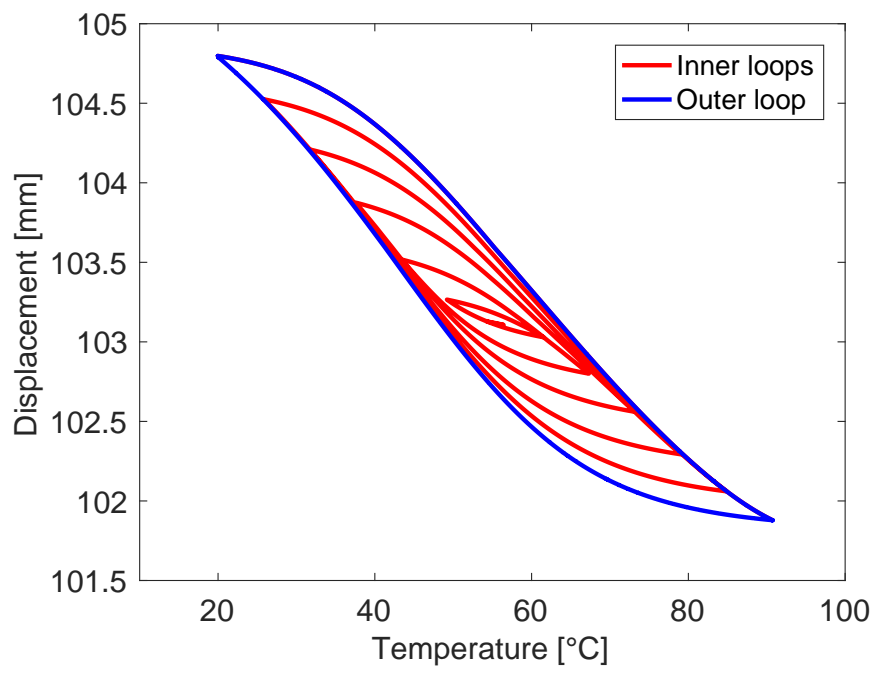

Fig. 1. Temperature-displacement hysteresis of a typical shape memory alloy wire-spring actuator.

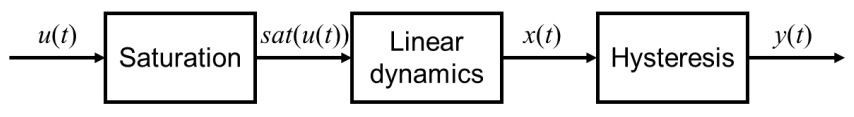

Fig. 2. Block diagram representation of model (1)-(3).

where $\bar{u}>0$. A block diagram representation of the model (1)-(3) is shown in Fig. 2.

Our goal is to develop a mathematical framework for designing a proportional integral (PI) controller for the system (1)-(2) that allows achieving some desired specification, e.g., stability with some desired exponential decay rate $\alpha<0$, in spite both the hysteresis in (2) and saturation nonlinearity in (3).

Let us define the tracking error $e(t)$ as follows:

$$
e(t)=y^{*}-y(t)
$$

where $y^{*}$ is a constant set-point to be tracked. Let us also define the integral of the tracking error $e_{i}$ as:

$$
e_{i}(t)=\int_{0}^{t} e(\tau) d \tau=\int_{0}^{t}\left(y^{*}-y(\tau)\right) d \tau
$$

Assumption 1: Hysteresis in (2) admits a time derivative in the following form:

$$
\dot{y}(t)=\dot{\Gamma}(c x(t))=\frac{d \Gamma(c x(t))}{d(c x(t))} c \dot{x}(t)=\sigma(t) c \dot{x}(t)
$$

where $\sigma(t)$ is assumed as a piecewise-continuous function of time.

Assumption 2:

$$
\exists \underline{\sigma}, \bar{\sigma} \in \mathbb{R}, \underline{\sigma} \times \bar{\sigma}>0: \underline{\sigma} \leq \sigma(t) \leq \bar{\sigma} \quad \forall t \geq 0
$$

Assumption 1 holds if we consider, e.g., a hysteresis model which can be represented by a Prandtl-Ishlinskii operator [23]. In this case, $\sigma(t)$ has to be interpreted as the slope of the hysteresis in the $x-y$ plane at time $t$. Assumption 
2 restricts our class of hysteresis models to those which exhibits a strictly monotonic behavior, such that it is possible to identify a finite set to which $\sigma(t)$ belongs for every $t$. As remarked in [23], the extrema of such a set represent maximum and minimum slope of the hysteresis, and can be identified from a simple input-output experiment. Therefore, the bounds on $\sigma(t)$ can be obtained without performing an identification of the complete hysteresis operator.

By differentiating (4)-(5) and accounting for (1) and Assumption 1, we have:

$$
\begin{aligned}
& \dot{e}_{i}(t)=e(t) \\
& \dot{e}(t)=-\sigma(t) c \dot{x}(t)
\end{aligned}
$$

Let us now define:

$$
\begin{aligned}
& z_{1}(t)=e(t) \\
& z_{2}(t)=\dot{x}(t)=A x(t)+b s a t(u(t))
\end{aligned}
$$

and let us propose the following PI law for (1):

$$
u(t)=k_{p} e(t)+k_{i} e_{i}(t)
$$

with gains $k_{p}$ and $k_{i}$ to be determined. By differentiating $z_{1}$ and $z_{2}$ in time, we can distinguish two cases: the first one corresponding to the linear region of the saturation function, the second one corresponding to the constant region.

\section{A. Linear region}

When the system is working in the linear region of the saturation function, (11) becomes:

$$
z_{2}(t)=A x(t)+b u(t)
$$

Hence, we obtain:

$$
\begin{aligned}
& \dot{z}_{1}(t)=\dot{e}(t) \\
& \dot{z}_{2}(t)=A \dot{x}(t)+b \dot{u}(t)
\end{aligned}
$$

By replacing (12) in (15) we obtain

$$
\begin{aligned}
& \dot{z}_{1}(t)=\dot{e}(t) \\
& \dot{z}_{2}(t)=A \dot{x}(t)+b k_{p} \dot{e}(t)+b k_{i} \dot{e}_{i}(t)
\end{aligned}
$$

By combining (8)-(9), (11) with (16)-(17), the following autonomous dynamic system is obtained:

$$
\left[\begin{array}{c}
\dot{z}_{1}(t) \\
\dot{z}_{2}(t)
\end{array}\right]=\left[\begin{array}{cc}
0 & -\sigma(t) c \\
b k_{i} & A-b k_{p} \sigma(t) c
\end{array}\right]\left[\begin{array}{c}
z_{1}(t) \\
z_{2}(t)
\end{array}\right]
$$

If the state matrix of the system (18) is made stable, by choosing properly the gains $k_{p}$ and $k_{i}$, then $z_{1}(t)=e(t)$ will converge to zero, meaning that at steady-state the tracking goal is achieved.

\section{B. Constant region}

When the system is working in the constant region of the saturation function, (11) becomes:

$$
z_{2}(t)=A x(t) \pm b \bar{u}
$$

Hence, the autonomous dynamic system which describes the evolution of $z_{1}(t)$ and $z_{2}(t)$ is given by:

$$
\left[\begin{array}{c}
\dot{z}_{1}(t) \\
\dot{z}_{2}(t)
\end{array}\right]=\left[\begin{array}{cc}
0 & -\sigma(t) c \\
0 & A
\end{array}\right]\left[\begin{array}{l}
z_{1}(t) \\
z_{2}(t)
\end{array}\right]
$$

which, as expected, does not depend on the controller gains $k_{p}$ and $k_{i}$. For the system (20), exponential stability cannot be obtained due to the existence of a null eigenvalue and, possibly, some positive ones in the matrix $A$. For this reason, the approach proposed in the following section aims at avoiding the constant region of the saturation function by employing a finite time stability specification.

\section{Control based on BMI With Finite time STABILITY}

First of all, let us recall the definition of finite time stability, as given by [26]:

Definition 1: The system $\dot{x}(t)=A x(t)$ is finite time stable (FTS) with respect to $\left(c_{1}, c_{2}, T, R\right)$, with $c_{2}>c_{1}$ and $R \succ 0$, if:

$$
x(0)^{T} R x(0) \leq c_{1} \Rightarrow x(t)^{T} R x(t)<c_{2} \quad \forall t \in[0, T]
$$

A condition for assessing finite time stability of an autonomous system is given by [28]:

Theorem 1: The system $\dot{x}(t)=A x(t)$ is FTS with respect to $\left(c_{1}, c_{2}, T, R\right)$ if, letting $\tilde{Q}=R^{-1 / 2} Q R^{-1 / 2}$, there exist scalars $\delta>0, \lambda>0$ and a matrix $Q \succ 0$ such that:

$$
\begin{gathered}
A \tilde{Q}+\tilde{Q} A^{T}-\delta \tilde{Q} \prec 0 \\
\lambda I \prec Q \prec I \\
{\left[\begin{array}{cc}
c_{2} e^{-\delta T} & \sqrt{c_{1}} \\
\sqrt{c_{1}} & \lambda
\end{array}\right] \succ 0}
\end{gathered}
$$

Let us augment the state of (18) by considering $z_{3}(t)=u(t)$, thus obtaining:

$$
\left[\begin{array}{c}
\dot{z}_{1}(t) \\
\dot{z}_{2}(t) \\
\dot{z}_{3}(t)
\end{array}\right]=\left[\begin{array}{ccc}
0 & -\sigma(t) c & 0 \\
b k_{i} & A-b k_{p} \sigma(t) c & 0 \\
k_{i} & -k_{p} \sigma(t) c & 0
\end{array}\right]\left[\begin{array}{c}
z_{1}(t) \\
z_{2}(t) \\
z_{3}(t)
\end{array}\right]
$$

If bounds for the state vector $x(t)$ are known, then it is possible to obtain bounds for the tracking error $e(t)$, i.e., a bound on $z_{1}(t)$. Moreover, by choosing properly the initial value for $e_{i}(t)$ as $e_{i}(0)=-k_{p} e_{0} / k_{i}, z_{3}(0)=0$ is obtained along with $z_{2}(0)=A x(0)$, such that a bound for $z_{2}(0)$ can be computed as well. Hence, it is possible to write (21) as:

$$
\begin{aligned}
& {\left[\begin{array}{l}
z_{1}(0) \\
z_{2}(0)
\end{array}\right]^{T} R\left[\begin{array}{l}
z_{1}(0) \\
z_{2}(0)
\end{array}\right] \leq c_{1} \Rightarrow} \\
& \Rightarrow\left[\begin{array}{l}
z_{1}(t) \\
z_{2}(t)
\end{array}\right]^{T} R\left[\begin{array}{l}
z_{1}(t) \\
z_{2}(t)
\end{array}\right]+z_{3}(t)^{T} S z_{3}(t)<c_{2} \quad \forall t \in[0, T]
\end{aligned}
$$

and choose $S$ and $c_{2}$ to ensure that the control input $u(t)$ will not saturate during the time interval $[0, T]$, where $T$ is chosen big enough for the states $z_{1}$ and $z_{2}$ to converge in a neighbourhood of the origin. 
Although Theorem 1 could be used to assess FTS of (25), it is worth noting that a lot of conservativeness is involved in the fact that: i) $z_{3}(0)=0$ is not taken into account; and ii) the state matrix in (25) has a particular structure, which could be exploited taking into account that the subsystem corresponding to the states $z_{1}(t)$ and $z_{2}(t)$ is made exponentially stable by design.

Theorem 2: The following statements are true:

- the system $\dot{x}_{1}(t)=A_{1} x_{1}(t)$ is quadratically stable with exponential decay rate $\alpha<0$;

- the system:

$$
\dot{x}(t)=\left[\begin{array}{c}
\dot{x}_{1}(t) \\
\dot{x}_{2}(t)
\end{array}\right]=\left[\begin{array}{cc}
A_{1} & 0 \\
A_{2} & 0
\end{array}\right]\left[\begin{array}{l}
x_{1}(t) \\
x_{2}(t)
\end{array}\right]=A x(t)
$$

is FTS with respect to $\left(c_{1}, c_{2}, T, R\right)$ with $c_{2}>c_{1}, R$ structured as $R=\operatorname{diag}\left(R_{1}, R_{2}\right)$, and $x_{2}(0)=0$;

if, letting:

$\tilde{Q}=\left[\begin{array}{ll}\tilde{Q}_{11} & \tilde{Q}_{12} \\ \tilde{Q}_{12}^{T} & \tilde{Q}_{22}\end{array}\right]=R^{1 / 2}\left[\begin{array}{cc}Q_{11} & Q_{12} \\ Q_{12}^{T} & Q_{22}\end{array}\right] R^{1 / 2}=R^{1 / 2} Q R^{1 / 2}$

there exist scalars $\delta>0, \lambda>0$ and a matrix $Q \succ 0$ such that:

$$
A_{1}^{T} \tilde{Q}_{11}+\tilde{Q}_{11} A_{1}-\alpha \tilde{Q}_{11} \prec 0
$$

$\left[\begin{array}{cc}(\alpha-\delta) \tilde{Q}_{11}+\operatorname{sym}\left\{\tilde{Q}_{12} A_{2}\right\} & A_{1}^{T} \tilde{Q}_{12}+A_{2}^{T} \tilde{Q}_{22}-\delta \tilde{Q}_{12} \\ \tilde{Q}_{12}^{T} A_{1}+\tilde{Q}_{22} A_{2}-\delta \tilde{Q}_{12}^{T} & -\delta \tilde{Q}_{22}\end{array}\right] \prec 0$

$$
\begin{gathered}
\lambda I \prec Q \\
Q_{11} \prec I \\
c_{1}<\lambda c_{2} e^{-\delta T}
\end{gathered}
$$

with $\operatorname{sym}\{X\}=X+X^{T}$.

Proof: Let $V(x(t))$ be defined as:

$$
V(x(t))=x(t)^{T} \tilde{Q} x(t)=V_{1}\left(x_{1}(t)\right)+2 V_{12}(x(t))+V_{2}\left(x_{2}(t)\right)
$$

with:

$$
\begin{aligned}
& V_{1}\left(x_{1}(t)\right)=x_{1}(t)^{T} \tilde{Q}_{11} x_{1}(t) \\
& V_{12}(x(t))=x_{1}(t)^{T} \tilde{Q}_{12} x_{2}(t) \\
& V_{2}\left(x_{2}(t)\right)=x_{2}(t)^{T} \tilde{Q}_{22} x_{2}(t)
\end{aligned}
$$

and note that (29) implies:

$$
\dot{V}_{1}\left(x_{1}(t)\right)<\alpha V_{1}\left(x_{1}(t)\right)
$$

while (30) corresponds to:

$$
2 \dot{V}_{12}(x(t))+\dot{V}_{2}\left(x_{2}(t)\right)<\delta V(x(t))-\alpha V_{1}\left(x_{1}(t)\right)
$$

which, in view of (34), leads to:

$$
\dot{V}(x(t))<\delta V(x(t)) \rightarrow V(x(t))<V(x(0)) e^{\delta t}
$$

and, taking into account that $x_{2}(0)=0$ :

$$
V(x(t))<V_{1}\left(x_{1}(0)\right) e^{\delta t}
$$

By considering that:

$$
V(x(t))=x(t)^{T} R^{1 / 2} Q R^{1 / 2} x(t) \geq \lambda_{\min }(Q) x(t)^{T} R x(t)
$$

and:

$$
\begin{aligned}
V_{1}\left(x_{1}(0)\right) e^{\delta t} & =x_{1}(0)^{T} R_{1}^{1 / 2} Q_{11} R_{1}^{1 / 2} x_{1}(0) e^{\delta t} \\
& \leq \lambda_{\max }\left(Q_{11}\right) x_{1}(0)^{T} R_{1} x_{1}(0) e^{\delta t} \\
& \leq \lambda_{\max }\left(Q_{11}\right) c_{1} e^{\delta T}
\end{aligned}
$$

the following is obtained:

$$
x(t)^{T} R x(t)<\frac{\lambda_{\max }\left(Q_{11}\right) c_{1} e^{\delta T}}{\lambda_{\min }(Q)}
$$

for which a condition for FTS of (27) with respect to $\left(c_{1}, c_{2}, T, R\right)$ is obtained as:

$$
\frac{c_{1}}{\lambda_{\min }(Q)}<\frac{c_{2} e^{-\delta T}}{\lambda_{\max }\left(Q_{11}\right)}
$$

Then, it is easy to check that, by requiring $\lambda_{\min }(Q)>\lambda$ and $\lambda_{\text {max }}\left(Q_{11}\right)<1$, i.e. (31)-(32), (41) leads to (33), which completes the proof.

Let us now rewrite the state matrix in (25) as:

$$
\left[\begin{array}{c}
\dot{z}_{1}(t) \\
\dot{z}_{2}(t) \\
\dot{z}_{3}(t)
\end{array}\right]=\left[\begin{array}{cc}
A_{11}(t)+B_{1} K C_{1}(t) & 0 \\
K C_{1}(t) & 0
\end{array}\right]\left[\begin{array}{c}
z_{1}(t) \\
z_{2}(t) \\
z_{3}(t)
\end{array}\right]
$$

with:

$$
\begin{gathered}
A_{11}(t)=\left[\begin{array}{cc}
0 & -\sigma(t) c \\
0 & A
\end{array}\right] \quad B_{1}=\left[\begin{array}{l}
0 \\
b
\end{array}\right] \\
K=\left[\begin{array}{ll}
k_{i} & k_{p}
\end{array}\right] \quad C_{1}(t)=\left[\begin{array}{cc}
1 & 0 \\
0 & -\sigma(t) c
\end{array}\right]
\end{gathered}
$$

Hence, (29)-(30) become:

$$
\begin{gathered}
\operatorname{sym}\left\{\tilde{Q}_{11}\left(A_{11}(t)+B_{1} K C_{1}(t)\right)\right\}-\alpha \tilde{Q}_{11} \prec 0 \\
{\left[\begin{array}{cc}
(\alpha-\delta) \tilde{Q}_{11}+\operatorname{sym}\left\{\tilde{Q}_{12} K C_{1}(t)\right\} & \text { (43) } \\
\tilde{Q}_{12}^{T}\left(A_{11}(t)+B_{1} K C_{1}(t)\right)+\tilde{Q}_{22} K C_{1}(t)-\delta \tilde{Q}_{12}^{T} & -\delta \tilde{Q}_{22}
\end{array}\right] \prec 0}
\end{gathered}
$$

which, in conjuction with the existence of bounds on $\sigma(t)$, as in (7), are satisfied if:

$$
\begin{gathered}
\operatorname{sym}\left\{\tilde{Q}_{11}\left(\underline{A_{11}}+B_{1} K \underline{C_{1}}\right)\right\}-\alpha \tilde{Q}_{11} \prec 0 \\
\operatorname{sym}\left\{\tilde{Q}_{11}\left(\overline{A_{11}}+B_{1} K \overline{C_{1}}\right)\right\}-\alpha \tilde{Q}_{11} \prec 0 \\
{\left[\begin{array}{cc}
(\alpha-\delta) \tilde{Q}_{11}+\operatorname{sym}\left\{\tilde{Q}_{12} K C_{1}\right\} & * \\
\tilde{Q}_{12}^{T}\left(\underline{A_{11}}+B_{1} K \underline{C_{1}}\right)+\tilde{Q}_{22} K \underline{C_{1}}-\delta \tilde{Q}_{12}^{T} & -\delta \tilde{Q}_{22}
\end{array}\right] \prec 0} \\
{\left[\begin{array}{cc}
(\alpha-\delta) & \tilde{Q}_{11}+\operatorname{sym}\left\{\tilde{Q}_{12} K \overline{C_{1}}\right\} \\
\tilde{Q}_{12}^{T}\left(\overline{A_{11}}+B_{1} K \overline{C_{1}}\right)+\tilde{Q}_{22} K \overline{C_{1}}-\delta \tilde{Q}_{12}^{T} & -\delta \tilde{Q}_{22}
\end{array}\right] \prec 0}
\end{gathered}
$$

where:

$$
\begin{array}{ll}
\underline{A_{11}}=\left[\begin{array}{cc}
0 & -\underline{\sigma} c \\
0 & \bar{A}
\end{array}\right] & \underline{C_{1}}=\left[\begin{array}{cc}
1 & 0 \\
0 & -\underline{\sigma} c
\end{array}\right] \\
\overline{A_{11}}=\left[\begin{array}{cc}
0 & -\bar{\sigma} c \\
0 & A
\end{array}\right] & \overline{C_{1}}=\left[\begin{array}{cc}
1 & 0 \\
0 & -\bar{\sigma} c
\end{array}\right]
\end{array}
$$




\section{ACADEMIC EXAMPLE}

Let us consider the system:

$$
\begin{aligned}
& \dot{x}(t)=0.5 x(t)+10 \text { sat }(u(t)) \\
& y(t)=\Gamma(x(t))
\end{aligned}
$$

with $\bar{u}=10, \underline{\sigma}=-0.6, \bar{\sigma}=-0.3$. A hysteretic model based on a standard play operator superimposed to a line is chosen [23], in such a way the hysteresis slope switches continuously between $\underline{\sigma}$ and $\bar{\sigma}$ depending on the specific loading/unloading branch. Bounds on $z_{1}(0), z_{2}(0)$ are chosen as follows: $\left|z_{1}(0)\right| \leq z_{1}^{\max }=1,\left|z_{2}(0)\right| \leq z_{2}^{\max }=15 . R$ is chosen as $R=\operatorname{diag}(1,0.1)$, hence $c_{1}=\left(z_{1}^{\max }\right)^{2}+0.1\left(z_{2}^{\max }\right)^{2}=23.5$. The scalar $S$ is chosen as $S=1$, such that the constraint on the input value can be expressed as $c_{2}=\bar{u}^{2}=100$. The desired decay rate is set as $\alpha=-0.2$, while $T=\ln (0.05) / \alpha \cong 15 \mathrm{~s}$ such that until the Lyapunov function $V_{1}\left(z_{1}(t), z_{2}(t)\right)$ has converged to $5 \%$ of the initial value $V_{1}\left(z_{1}(0), z_{2}(0)\right)$, the input will not saturate for sure. Finally, a value of $\delta=0.01$ is selected.

Applying Theorem 2 using the YALMIP toolbox [29] with PENBMI solver [30], we obtain:

$$
Q=\left(\begin{array}{ccc}
0.4479 & 0.1383 & 0.0366 \\
0.1383 & 0.9608 & -0.0879 \\
0.0366 & -0.0879 & 0.3115
\end{array}\right)
$$

and $\lambda=0.273, k_{i}=-0.1571, k_{p}=-0.2693$.

Response of both system state $x(t)$ and output $y(t)$ is shown in Fig. 3 for a simulation lasting $400 s$, in which the system is required to follow a multiple step tracking reference. It can be noted how convergence to the set-point with the desired decay rate is ensured. Fig. 4 shows a plot of the relation between $x(t)$ and $y(t)$ during the simulation, which proves that the output equation changes due to the hysteretic behavior. States $z_{1}(t)$ and $z_{2}(t)$ are represented in Fig. 5, while $z_{3}(t)$ is reported in Fig. 6. Note that $z_{1}(t)$ and $z_{2}(t)$ converge always to zero at steady-state, while the control input $z_{3}(t)$ remains always within the given bounds.

\section{CONCLUSION}

In this paper, a robust control strategy to deal with output regulation of a class of nonlinear dynamic systems has been presented. The class of considered systems, consisting of a linear dynamics cascaded with a hysteresis operator and subject to control input saturation, occurs commonly when dealing with smart material actuator systems. Due to a reformulation of the hysteretic system as a LPV model, it is possible to address the design of robust, inversionfree PI control laws by means of BMI conditions. To deal with control input saturation, a set of BMI has been developed based on the theory of finite time stability. The BMI conditions ensure that the control input of the closed loop system never exceeds a given saturation limits while providing, at the same time, set-point tracking with a desired exponential decay rate. The structure of the system has also been taken into account to find a set of novel BMI with
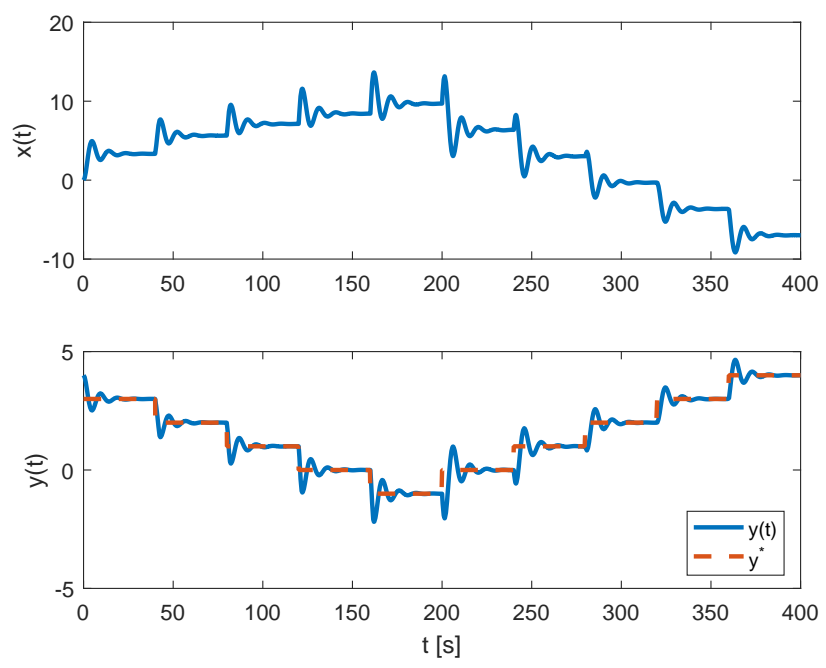

Fig. 3. Plot of $x(t)$ and $y(t)$.

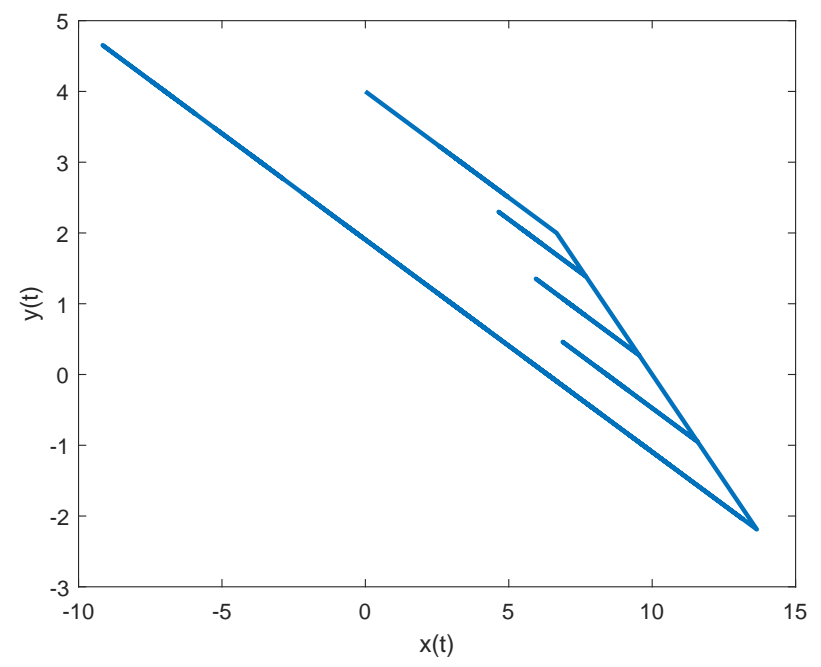

Fig. 4. Hysteresis plot of the relation $x(t)-y(t)$.

reduced conservatism. Effectiveness of the method has been proved with a simulation case of study.

In future works, the developed method will be extended to a larger class of dynamic systems, involving, e.g., multiinput multi-output dynamics affected by output hysteresis and input saturation on different channels, disturbances and parametric uncertainties. In addition, alternative formulations which allow further reduction of the conservativeness of the results will be investigated. Experimental validation of the resulting control laws on shape memory alloy actuators will be also performed.

\section{REFERENCES}

[1] M. V. Gandhi and B. Thompson, Smart materials and structures. Springer Science \& Business Media, 1992.

[2] G. Rizzello, L. Riccardi, D. Naso, B. Turchiano, and S. Seelecke, "An overview on innovative mechatronic actuators based on smart materials," in AFRICON, 2017 IEEE. IEEE, 2017, pp. 450-455. 

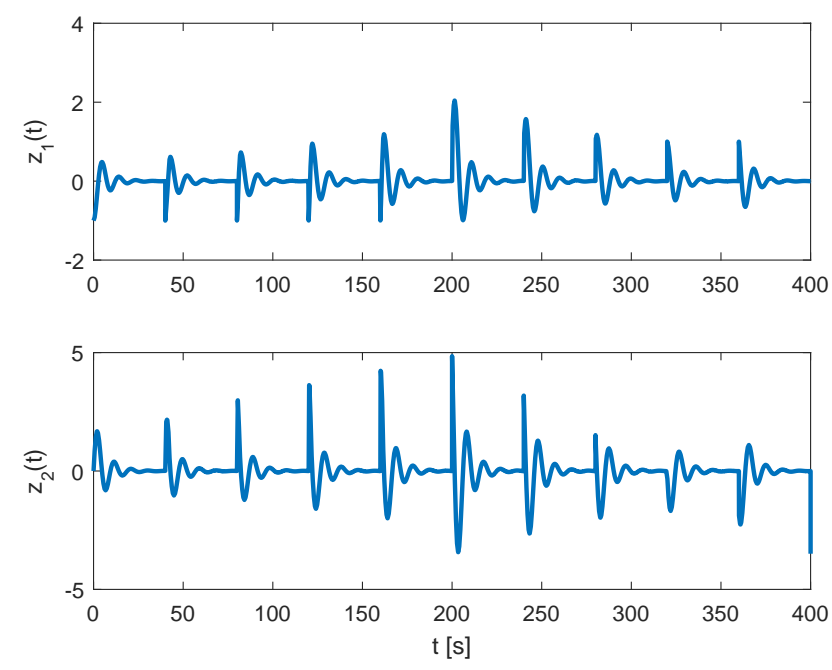

Fig. 5. Plot of $z_{1}(t)=e(t)$ and $z_{2}(t)=\dot{x}(t)$.

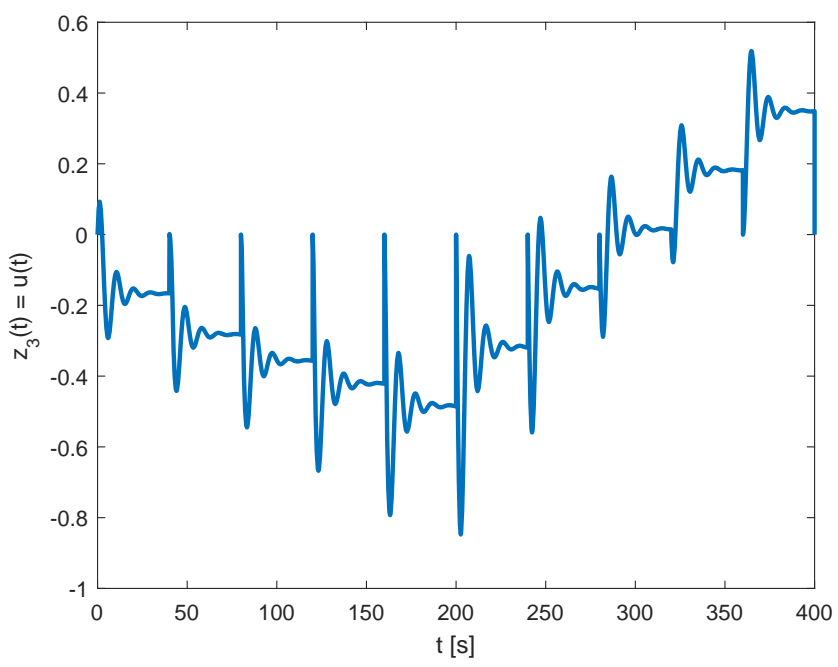

Fig. 6. Plot of $z_{3}(t)=u(t)$.

[3] X. Tan, "Control of smart actuators," Ph.D. dissertation, University of Maryland, 2002.

[4] M. Al Janaideh, S. Rakheja, and C.-Y. Su, "An analytical generalized Prandtl-Ishlinskii model inversion for hysteresis compensation in micropositioning control," IEEE/ASME Transactions on mechatronics, vol. 16, no. 4, pp. 734-744, 2011.

[5] R. V. Iyer and X. Tan, "Control of hysteretic systems through inverse compensation," IEEE Control Systems, vol. 29, no. 1, pp. 83-99, 2009.

[6] L. Riccardi, D. Naso, B. Turchiano, and H. Janocha, "Adaptive control of positioning systems with hysteresis based on magnetic shape memory alloys," IEEE Transactions on Control Systems Technology, vol. 21, no. 6, pp. 2011-2023, 2013.

[7] Y.-J. Liu, S. Tong, C. P. Chen, and D.-J. Li, "Neural controller designbased adaptive control for nonlinear MIMO systems with unknown hysteresis inputs," IEEE transactions on cybernetics, vol. 46, no. 1, pp. 9-19, 2016.

[8] R. B. Gorbet, K. A. Morris, and D. W. Wang, "Passivity-based stability and control of hysteresis in smart actuators," IEEE Transactions on control systems technology, vol. 9, no. 1, pp. 5-16, 2001.

[9] B. Jayawardhana, R. Ouyang, and V. Andrieu, "Stability of systems with the Duhem hysteresis operator: The dissipativity approach," Automatica, vol. 48, no. 10, pp. 2657-2662, 2012.

[10] H. Logemann, E. P. Ryan, and I. Shvartsman, "Integral control of infinite-dimensional systems in the presence of hysteresis: an inputoutput approach," ESAIM: Control, Optimisation and Calculus of Variations, vol. 13, no. 3, pp. 458-483, 2007.

[11] A. Esbrook, X. Tan, and H. K. Khalil, "Inversion-free stabilization and regulation of systems with hysteresis via integral action," Automatica, vol. 50, no. 4, pp. 1017-1025, 2014

[12] B. Jayawardhana, H. Logemann, and E. P. Ryan, "PID control of second-order systems with hysteresis," International Journal of Control, vol. 81, no. 8, pp. 1331-1342, 2008.

[13] S. Valadkhan, K. Morris, and A. Khajepour, "Stability and robust position control of hysteretic systems," International Journal of Robust and Nonlinear Control, vol. 20, no. 4, pp. 460-471, 2010.

[14] A. Esbrook, X. Tan, and H. K. Khalil, "Control of systems with hysteresis via servocompensation and its application to nanopositioning," IEEE Transactions on Control Systems Technology, vol. 21, no. 3, pp. 725-738, 2013.

[15] M. Edardar, X. Tan, and H. K. Khalil, "Sliding-mode tracking control of piezo-actuated nanopositioners," in American Control Conference (ACC), 2012. IEEE, 2012, pp. 3825-3830.

[16] S. Miyamoto and G. Vinnicombe, "Robust control of plants with saturation nonlinearity based on coprime factor representations," in Decision and Control, 1996., Proceedings of the 35th IEEE Conference on, vol. 3. IEEE, 1996, pp. 2838-2840.

[17] A. Saberi, Z. Lin, and A. R. Teel, "Control of linear systems with saturating actuators," IEEE Transactions on Automatic Control, vol. 41, no. 3, pp. 368-378, 1996.

[18] T. Hu and Z. Lin, Control systems with actuator saturation: analysis and design. Springer Science \& Business Media, 2001.

[19] S. Tarbouriech, G. Garcia, J. M. G. da Silva Jr, and I. Queinnec, Stability and stabilization of linear systems with saturating actuators. Springer Science \& Business Media, 2011.

[20] J. Jayender, R. Patel, S. Nikumb, and M. Ostojic, " $h_{\infty}$ loop shaping controller for shape memory alloy actuators," in Decision and Control, 2005 and 2005 European Control Conference. CDC-ECC'05. 44th IEEE Conference on. IEEE, 2005, pp. 653-658.

[21] M. Al Janaideh and D. S. Bernstein, "Inversion-free adaptive control of uncertain systems with shape-memory-alloy actuation," in American Control Conference (ACC), 2013. IEEE, 2013, pp. 3579-3584.

[22] L. F. Toledo, Z. G. Joey, J. M. Oxoby, Y. Chen, and N. O. PérezArancibia, "System identification of a NiTi-based SMA actuator using a modified Preisach model and adaptive control," in American Control Conference (ACC), 2017. IEEE, 2017, pp. 183-190.

[23] L. Riccardi, D. Naso, B. Turchiano, and H. Janocha, "Design of linear feedback controllers for dynamic systems with hysteresis," IEEE Transactions on Control Systems Technology, vol. 22, no. 4, pp. 12681280, 2014

[24] D. Rotondo, V. Puig, F. Nejjari, and M. Witczak, "Automated generation and comparison of Takagi-Sugeno and polytopic quasi-LPV models," Fuzzy Sets and Systems, vol. 277, pp. 44-64, 2015.

[25] D. Rotondo, Advances in gain-scheduling and fault tolerant control techniques. Springer, 2017.

[26] F. Amato, M. Ariola, and P. Dorato, "Finite-time control of linear systems subject to parametric uncertainties and disturbances," Automatica, vol. 37, no. 9, pp. 1459-1463, 2001.

[27] D. Rotondo, F. Nejjari, and V. Puig, "Dilated LMI characterization for the robust finite time control of discrete-time uncertain linear systems," Automatica, vol. 63, pp. 16-20, 2016.

[28] F. Amato, M. Ariola, M. Carbone, and C. Cosentino, "Finite-time control of linear systems: a survey," in Current trends in nonlinear systems and control. Springer, 2006, pp. 195-213.

[29] J. Lofberg, "Yalmip: A toolbox for modeling and optimization in MATLAB," in Computer Aided Control Systems Design, 2004 IEEE International Symposium on. IEEE, 2004, pp. 284-289.

[30] D. Henrion, J. Lofberg, M. Kocvara, and M. Stingl, "Solving polynomial static output feedback problems with PENBMI," in Decision and Control, 2005 and 2005 European Control Conference. CDC-ECC'05. 44th IEEE Conference on. IEEE, 2005, pp. 7581-7586. 